\title{
Comparison of Common Methods from Intertwined Application in Image Processing
}

\author{
Seong-Yoon Shin, Hyun-Chang Lee and Yang-Won Rhee, Member, KIMICS
}

\begin{abstract}
Image processing operations like smoothing and edge detection, and many more are very widely used in areas like Computer Vision. We classify the image processing domain as seven branches-image acquirement and output, image coding and compression, image enhancement and restoration, image transformation, image segmentation, image description, and image recognition and description. We implemented algorithms of gaussian smoothing, laplace sharpening, image contrast effect, image black and white effect, image fog effect, image bright and dark effect, image median filter, and canny edge detection. Such experimental results show the figures respectively.
\end{abstract}

Index Terms - image processing, gaussian smoothing, laplace sharpening, canny edge detection

\section{INTRODUCTION}

Digital image processing is the use of computer algorithms to perform image processing on digital images. As a subcategory or field of digital signal processing, digital image processing has many advantages over analog image processing. It allows a much wider range of algorithms to be applied to the input data and can avoid problems such as the build-up of noise and signal distortion during processing. Since images are defined over two dimensions(perhaps more) digital image processing may be modeled in the form of Multidimensional Systems.

Hitherto the computer already widely used around the world, and the image processing also stretch to daily work. From the perspective of computer, an image is usually interpreted as a two dimensional array of brightness values, and then reduced to a series of numbers that can be manipulated by the computer. Each number representing the brightness value of the image at a particular location is called a pixel. Once the image has been digitized like this, there are at least three basic operations that can be performed on it in the computer [1]. For a point operation, a pixel value in the output image depends on a single pixel value in the input image. For local operations, several neighboring pixels in the input image determine the value of an output image pixel. In a

\footnotetext{
Manuscript received July 1, 2010; accepted July 1, 2010.

Seong-Yoon and Yang-Won Rhee is with the Dept. of Computer Information Eng., Kunsan Natl. Univ., Kunsan, Korea. (Email: s3397220, ywhrhee@kunsan.ac.kr)

Hyun-Chang Lee(Corresponding Author) is with the Div. of Inf. and Elec. Commerce, Wonkwang Univ., Iksan, Korea. (Email: hclglory@wku.ac.kr)
}

global operation, all of the input image pixels contribute to an output image pixel value. These operations, taken singly or in combination, are the means by which the image is enhanced, restored, or compressed.

\section{IMAGE PROCESSING DOMAIN}

\section{A. Image acquirement and oupput}

This is the main study current in the image processing domain recently who purposely transforms the analog image signal into other type which can be accepted by the computer, and then take them present as the practical form. The ubiquitous acquiring devices are digital camera, scanner; while the usual output devices are the print, slide, television or movie screen and so on.

\section{B. Image coding and compression}

The purpose of image coding and compression is representing an image by fewer numbers, at the same time minimizing the degradation of the information contained in the image. This part is important because of the large quantities of digital imagery that are sent electronically and stored. Digital high definition television relies heavily on image compression to enable transmission and display of large format color images. Once the image is compressed for storage or transmission, it must be uncompressed for use, by the inverse of the compression operations. There is a tradeoff between the amount of compression and the quality of the uncompressed image. High compression rates are acceptable with television images for example. However, the high image quality must be preserved, only compression rates as low as three to four may be acceptable. This conflict develops fiercer before the emergence for the computer network.

\section{Image enhancement and restoration}

The aim of enhancement and restoration are to improve the image. An image is enhanced when it is modified so that the information it contains is more clearly evident. But enhancement can also include making the image more visually appealing. The example is noise smoothing, sharpening and contrast. The restoration is unlike enhancement, knowledge of how the image was formed is used in an attempt to retrieve the ideal image. Any image forming system is not perfect, and will introduce artifacts into the final image that would not be present in an ideal image. A point spread function, called the filter, can be 
constructed that undoes the blurring. By imaging the blurred image with the filter point spread function, the restored image results. The filter point spread function is spread out more than the blurring point spread function, bringing more pixels into the averaging process. This is an example of a global operation, since perhaps all of the pixels of the blurred image can contribute to the value of a single pixel in the restored image. This type of deblurring is called inverse filtering, and is sensitive to the presence of noise in the blurred image. By modifying the deblurring filter according to the properties of the noise, performance can be improved.

\section{Image transformation}

The purpose of image transformation is changing from spatial domain processing to transform processing, in order to reduce the computer work then get the more effective results. The common methods are Fourier transform, Walsh transform, discrete cosine transform and so on.

\section{E. Image segmentation}

The purpose of image segmentation pick up the meaning features, which include of the contour or the area, out of the image, then get further recognize, analysis and comprehensive. This aspect mainly consists of edge detection and outline tracking. Recently, the research is delving into deeper and deeper.

\section{F. Image description}

This part supposed to be the necessary foundation of image recognize. As the upper mention, regarding the image as two dimensional array is precondition. Then describe the practical texture feature with two dimensional texture feature description, others like volume description, surface area description and generalized cylinder description are also used widely.

\section{Image recognition and detection}

This part is mainly segment image and pick up features out of the image which undergone some practical processing. It is better for computer dealing with the image effectually and effectively, as determining the existent of targets and then locating them.

Over all, image processing is an active area of research in such diverse fields as medicine, astronomy, microscopy, seismology, defense, industrial quality control, and the publication and entertainment industries. The concept of an image has expanded to include three dimensional data sets, and even four dimensional volume time data sets. An example of the latter is a volume image of a beating heart, obtainable with x-ray computed tomography. CT, PET, single-photon emission on computed tomography, MRI, ultrasound, SAR, Confocal microscopy, scanning tunneling microscopy, atomic force microscopy, and other modalities have been developed to provide digitized images directly. Digital images are widely available from the Internet, CD-ROM, and inexpensive charge-coupleddevice (CCD) camera, scanner, and frame grabber. Software for manipulating images is also widely available. In summary, the image processing has already infiltrated into our lives everywhere, getting the ropes become a maneuver for impelling further research exactly. So it is necessary for study the image processing and benefit in the end.

\section{COMMON BASIC IMAGE PROCESSING METHDS}

The following information comes from [11]. The image through processing is suitable for the particular application. This means that the image processing technique have to intertwine with the application, in the different environments and circumstances the method and purpose is absolutely different, even the method convenience for user may not suit the computer recognize.

The common image processing method treat the image as the array of pixels, and every pixel with its eight neighbors who directly around it form a square neighbor domain, sometimes may be more than that such as $5 \times 5$, or $7 \times 7$, and then adopt the convolution and template for every possible pixel value and then divide a attenuation factor, restrict the result in the area between 0 and 255, while the original and processed value record respectively. During the whole procedure, the result sole exist and represent the processing without changing the original pixel.

\section{A. Gaussian Smoothing}

The Gaussian smoothing in two dimensional distributions is non-zero everywhere, which would require an infinitely large convolution kernel, but in practice it is effectively zero more than about three standard deviations form the mean, and so it is usually truncate the kernel at this point [2].

$$
G(x, y)=\frac{1}{2 \pi \sigma^{2}} e^{-\frac{x^{2}+y^{2}}{2 \sigma^{2}}}
$$

in two dimensions

The Gaussian equation

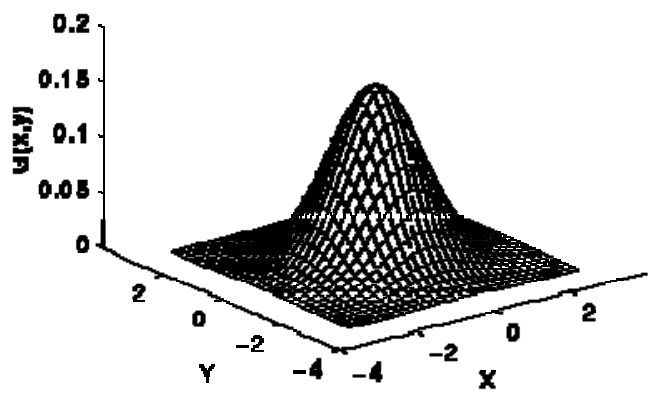

Fig.1 Gaussian equation and distribution 
The image through processing is suitable for the particular application. This means that the image processing technique have to intertwine with the application, in the different environments and circumstances the method and purpose is absolutely different, even the method convenience for user may not suit the computer recognize.

The distribution with mean $(0,0)$ and $\sigma=1.0$. And its suitable integer-valued convolution kernel approximates a Gaussian with $\sigma$ of 1.0. The following figure is the discrete approximation to Gaussian function with $\sigma=1.0$ :

$$
\left[\begin{array}{ccccc}
1 & 4 & 7 & 4 & 1 \\
4 & 16 & 26 & 16 & 4 \\
7 & 26 & 41 & 26 & 7 \\
4 & 16 & 26 & 16 & 4 \\
1 & 4 & 7 & 4 & 1
\end{array}\right] / 273
$$

But at here, just one step processing for smoothing, we simplify it further; just adopt a $3 \times 3$ window is enough:

$$
\left[\begin{array}{lll}
1 & 2 & 1 \\
2 & 4 & 2 \\
1 & 2 & 1
\end{array}\right] / 16
$$

If the other pixels in the neighbor area hard to the particular one, its weight will larger than the rest distant to the center, so that the result can obtain the general gray distribution feature besides blurring the details. And this trait is also the main advantage of the Gaussian smoothing method compared with the others. However, the Gaussian smoothing still has a fatal drawback that will degenerate the definition of the image while eliminating the noise points. Therefore, under the practical situation, Gaussian smoothing is a simple and effectual approach that can fast smooth the image.

\section{B. Laplace Sharpening}

In contrast to the Gaussian smoothing who can eliminate the noise points and reduce the contrast, the Laplace sharpening can enhance the outline and details to make the image clear.

When using Gaussian smoothing, it usually shrink the gap between the pixels' gray value in the neighbor domain through averaging. So in the Laplace sharpening, we just adverse the method and boost the contrast of image. In other words, only enlarge the gap between the particular pixel gray value with the rest in the neighbor domain, and the areas having same gray value will not affect by sharpening.

This method always uses a Laplace operator to improve the image boundary. The Laplace operator stems from the second differential, and base on the difference calculation of pixels' gray value of neighbor domain. The basic idea is that when the center pixel's gray value is higher than its neighbor's average; heighten it further, and vice versa. In the end, will get the image with contrast enhanced [3].

This method always uses a Laplace operator to improve the image boundary. The Laplace operator stems from the second differential, and base on the difference calculation of pixels' gray value of neighbor domain. The basic idea is that when the center pixel's gray value is higher than its neighbor's average; heighten it further, and vice versa. Intheend, will get the image with contrast enhanced [3]. The following figure is Laplace equation and its discrete form:

$$
\begin{aligned}
& \nabla^{2} f(x, y)=\frac{\partial^{2} f}{\partial x^{2}}+\frac{\partial^{2} f}{\partial y^{2}} \text { The Laplace equation } \\
& \nabla^{2} f=[f(x+1, y)+f(x-1, y)+f(x, y+1)+f(x, y-1)]-4 f(x, y)
\end{aligned}
$$

The discrete form

And in practice, this method calculate the gradient on the direction from the center pixel to its eight neighbors, plus them up in order to determine their relationship, and then adjust the gray value by calculation result. The following figure is the simple template used in the implement:

$$
\left[\begin{array}{ccc}
-1 & -1 & -1 \\
-1 & 8 & -1 \\
-1 & -1 & -1
\end{array}\right]
$$

It is obvious that when the center pixel gray value is higher than the average of its neighbors', the result is positive, in contrast, is negative. So plus it on the original pixel, make the good performance of the center pixel.

Both the Gaussian smoothing and Laplace sharpening are belonging to the method of image enhancement and restoration. But as the Gaussian smoothing, the Laplace sharpening also has natural defect, any tiny noise point is sensitive, and its influence will be amplified in the result. Run over it, Laplace sharpening is still a fast and simple method to improve the image contrast depends on the proper situation.

\section{Image contrast effect}

Image filter is a other useful application of image processing that can make special good effect for the original image under the user's requirements. From the present, it seems as add an extra tier for artistic effect; while in reality, it focus on the relationship of the adjacent pixels, distinguish from the neighbor domain concept. Through particular mathematical calculation, it will get different results. The contrast effect is one of them.

In brief, the idea of contrast effect is just use every pixel's opposite gray value instead of the original one. Take RGB model for example: in RGB color space, every color has a point in Cartesian coordinate system, and its center symmetric point is reasonable the color opposite point. When the original RGB value is $(50,100,200)$, the contrast value is $(205,155,55)$ definitely.

\section{Image Black and White Effect}

From arctic aspect, the black and white image is full of nostalgic feelings, and tracing you back to the mesmerizing reminiscent of old school. Viewing from the technique, the black and white image highlight the 
elements such as the morphology, brightness, contrast, and texture, and make prepositional work for other complicated image processing.

Remembering the geometric representation of RGB color space, the RGB model is a cube in the Cartesian coordinate system, R, G, B are three coordinate axes. When all of them denote zero, it is the original point of coordinate, present black color; in opposition, all of them denote the maximum, the point represent white color. And the main diagonal line which connect the original with maximum point represent the gray scale. It means when the color change along this diagonal between black and white, also reveal the principle of gray image. In other words every point on the diagonal line has the same RGB value.

There are three frequently used black and white methods: the maximum method, which pixel's RGB value is equal to the maximum value among $\mathrm{R}, \mathrm{G}$, and $\mathrm{B}, \mathrm{MAX}$ $=(\mathrm{R}, \mathrm{G}, \mathrm{B})$; the average method, which every pixel's value is equal to the average value of $R, G, B, R=G=B$ $=(\mathrm{R}+\mathrm{G}+\mathrm{B}) / 3$; and the weighted average, which denote every RGB component a weight value, multiple it and calculate, get the result $R=G=B=(R * x+G * y+B *$ $\mathrm{z}) / 3$, where $\mathrm{x}, \mathrm{y}, \mathrm{z}$ all large than zero.

\section{E. Image Fog Effect}

The fog effect import random mechanism for pixel processing. This method seems cover the original image with a ground glass, make a atmosphere of misty. There are three usually used methods: the horizontal method, which transform a pixel value to another random pixel value in the horizontal direction, with random function who control the random pixel selection; the vertical method, compared with the horizontal method, just adjust the direction to vertical direction; and the combination method, which change the pixel to another one along the diagonal 45 degree direction.

\section{F. Image Bright and Dark Effect}

This procedure manipulates the pixel value only, need not to think about any relationship. So the implement is just directly plus or minus on the original value, the first mean bright it and the minus mean dark it. Image bright and dark effect is the fastest, simplest, and the most representative method depending on the pixel point directly.

\section{G. Image Median Filter}

The median filter is a non-linear digital filtering technique, often used to remove noise from image or other signals, especially treat speckle noise or salt and pepper noise. Different from the principle of other methods, such as Gaussian or Laplace smoothing which just average the noise spot effective on its neighborhoods, the median filter directly eliminate the isolate noise spot effective. To every suitable pixel, It first set up a template include its even number neighbors' pixels, and then arrange their values in numerical order, then pick the middle one as the pixel's value. Majority time, this approach can preserve the high quality of original image and get rid of the isolate noise expect of the edges.

\section{G. Canny Edge Detection}

The definition of edge is the boundary between an object and the background, and indicates the boundary between overlapping objects. This means if the edges in an image can be identified accurately, all of the objects can be located and basic properties can be measured further. And edge detection is a tool in image processing aim at identifying points in a digital image at which the image brightness changes sharply or more formally has discontinuities [4]. So the Canny edge detection is the approach can delineate the main contours of image.

Different from all of the above introduced one step methods, the Canny edge detection is a complicated one that consist of four processing phases generally.

The Canny algorithm uses an optimal edge detector based on a set of criteria which include finding the most edges by minimizing the error rate, marking edges as closely as possible to the actual edges to maximize localization, and marking edges only once when a single edge exists for minimal response [5]. According to Canny, the optimal filter that meets all three criteria above can be efficiently approximated using the first derivative of a Gaussian function. This part have introduced above, will not repeat here.

The first stage involves smoothing the image by convolving with a Gaussian filter. This is followed by finding the gradient of the image by feeding the smoothed image through a convolution operation with the derivative of the Gaussian in both the vertical and horizontal directions. The two dimensional convolution operations are described in the following equation.

$$
I^{\prime}(x, y)=g(k, l) \otimes I(x, y)=\sum_{k=-N}^{N} \sum_{l=-N}^{N} g(k, l) I(x-k, y-l)
$$

Where: $g(k, l)=$ convolutional kernel

$I(x, y)=$ original image

$I^{\prime}(x, y)=$ filtered image

$2 N+1=$ size of convolutional kernel

Both the Gaussian mask and its derivative are separable, allowing the two dimensional convolution operations to be simplified. The optimization is not limited to software implementation only, but applies to hardware implementation as well [6].

The non-maximal suppression stage finds the local maxima in the direction of the gradient, and suppresses all others, minimizing false edges. The local maxima found by comparing the pixel with its neighbors along the direction of the gradient. This helps to maintain the single pixel in edges before the final threshold stage.

Instead of using a single static threshold value for the entire image, the Canny algorithm introduced hysteresis threshold, which has some adaptively to the local content of the image. There are two threshold levels, $t_{h}$, high and 
$t_{l}$, low where $t_{h}>t_{l}$. Pixel values above the $t_{h}$ value are immediately classified as edges. By tracing the edge contour, neighboring pixels with gradient magnitude values less than $t_{h}$ can still be marked as edges as long as they are above $t_{l}$. This process alleviates problems associated with edge discontinuities by identifying strong edges, and preserving the relevant weak edges, in addition to maintaining some level of noise suppression [7]. While the results are desirable, the hysteresis stage slows the overall algorithm down considerably.

From the above description, the Canny edge detection is obvious a method that take simple algorithms as foundation, and take care of both the relationship between every pixel with its neighbors and every pixel's inimitable feature for the whole image, at the same time, also represent the advantage of black and white effect

\section{EXPERIMANTAL RESUTS}

Use the following image for experimentation

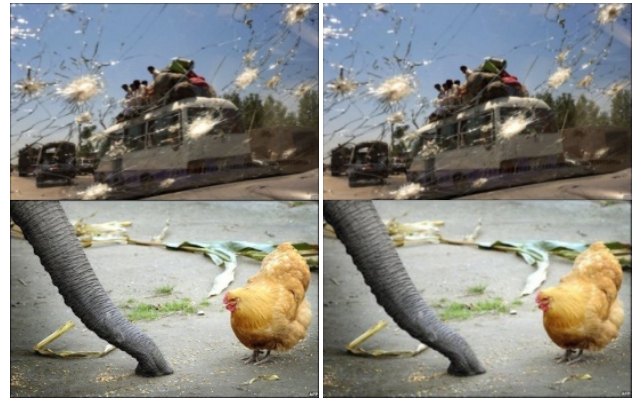

Fig.2 The source image and The soft effect

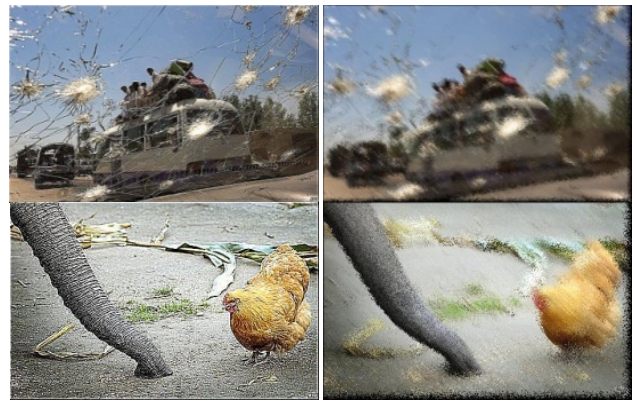

Fig.3. The sharp effect and The fog effect

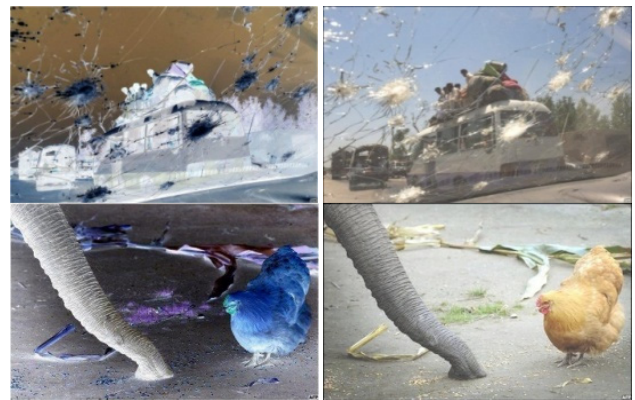

Fig.4 The contrast effect and The bright effect

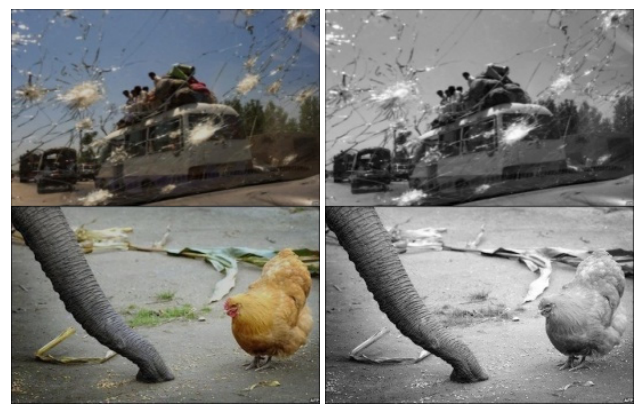

Fig.5 The dark effect and The black and white effect
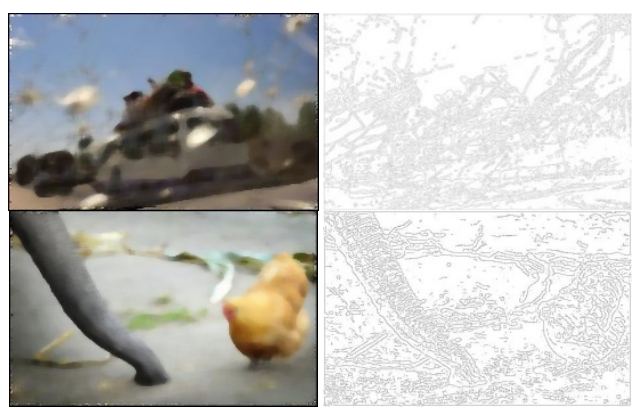

Fig.6 The median filter effect and The Canny effect

From the experimentation, every algorithm's feature has taken present obviously. Collecting their advantages means combining some of these methods in practice, but takes the Canny as example, its result can eliminate the star fracture, in other words, the noise points influence.

Therefore, these simple implement not only show us their special features, but also inspire us and force us further for their improvement.

\section{CONCLUSIONS}

We are investigated the implementation of the basic image processing algorithms. In additional, from the analysis of these image processing results, consider their theories and the actual role in the application, we can draw some conclusions as following. Almost of algorithms stand on the opposite sides, can get the different effect, but can't exist together. Majority time, when we want to get a kind of result, but take compensate for some others. From different aspects, such as cluster or boundary, single point or with its neighbor, all can get the particular result. But that doesn't mean they can cooperation well, sometimes even tackle each other. The physical mathematical calculation has to choose between the accuracy and timesaving.

The future we will try to develop more advanced algorithms. 


\section{REFERENCES}

[1] R. C. Gonzalez, and R. E. Woods, Digital Image Processing, Addison-Wesley Pub Co, 2002

[2] M. Basu, "Gaussian-Based Edge Detection Methods - A Survey", IEEE Trans. Systems, Man, and Cybernetics-Part C: Applications and Reviews, vol. 32, no. 3, pp. 252-60, Aug. 2002

[3] Zuo Fei, Wan Jinsen, and Liu Hang, Digital Image Processing Development and Implement, P452 P456, March 2007

[4] D. Marr, and E. Hildreth, "Theory of edge detection", Proc. Royal Society of London, Series B, Biological Sciences, vol. 207, no. 1167, pp. 187-217, Feb. 1980

[5] Canny, J., "A Computational Approach to Edge Detection", IEEE Trans. Pattern Analysis and Machine Intelligence, November 1986

[6] J. R. Parker, Algorithms for Image Processing and Computer Vision, P23 P29

[7] Hong Shan Neoh, and Asher Hazanchuk, "Adaptive Edge Detection for Real-Time Video Processing using FPGAs", Altera Corporation, 2004

[8] Chen Zhaoxue, and Shi Pengfei, "A fast method on locating and segmentation of vehicle plate base on gray image", Shanghai University of Science and Technology, 2006

[9] Liu Xinhai, and Fang Kangling, "A difference image algorithm for target positioning based on profile projection", School of Information Science \& Engineering, Wuhan University of Science and Technology, vol. 21, no. 7, July 2004

[10] Mei Lin, and Liu Feng, "A method of vehicle license plate location based on edge detection and vertical projection", Computer Science and Technology institute, Anhui University, 2009

[11] Shin S. Y., Rhee Y. W., Lee H. C., and Jung H. K., "Comparison of Common Image Processing Methods from Interwined Application," Proceedings International Conference of KIMICS 2010, pp. 225-228, June 2010

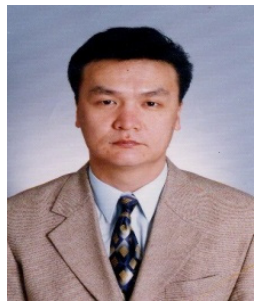

\section{Seong-Yoon Shin}

Received his M. S. and Ph. D degrees in Dept. of Computer Information Eng. from Kunsan Natl. Univ., Kunsan, Korea, in 1997 and 2003, respectively. From 2006 to present, he is a professor, Dept. of Computer Information Eng. in Kunsan Natl, Univ. His research interests include Image Processing, Computer Vision, and Virtual Reality.

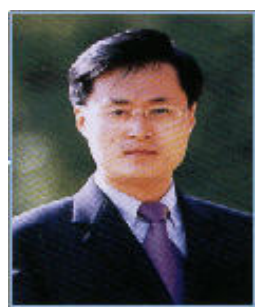

\section{Hyun-Chang Lee}

Received his M. S. and Ph. D degrees in Dept. of Computer Science from Hongik Univ., Seoul, Korea, in 1996 and 2001, respectively. From 2008 to present, he is a professor, Div. of Info. and Electronic Commerce. in Iksan. His research interests include Image Processing, Semantic web, Ontology, Ubiquitous Computing.

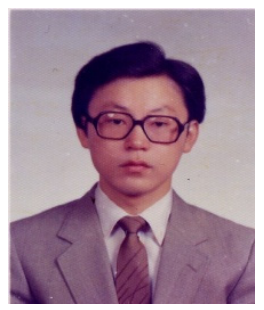

\section{Yang-Won Rhee}

Received his M. S. degree in Dept. of Computer Eng. From Yonsei Univ, Ph. D degree in Dept. of Computer Eng. From Soongsil Univ. From 1986 to present, he is a professor, Dept. of Computer Information Eng. in Kunsan Natl, Univ. His research interests include Pattern Recognition, Computer Vision, and Artificial Intelligence. 